\title{
Genetic and Environmental Sources of Variation in the Autogenous Chemical Defense of a Leaf Beetle
}

\author{
Y. Triponez • R. E. Naisbit • J. B. Jean-Denis •
}

M. Rahier • N. Alvarez

Published online: 24 October 2007

(C) Springer Science + Business Media, LLC 2007

The footnote to Table 4 does not correspond to the superscripts in the body of the table. The correct Table 4 appears below.

The online version of the original article can be found at doi:10.1007/s10886-007-9351-9.

Y. Triponez $(\bowtie) \cdot$ R. E. Naisbit $\cdot$ M. Rahier $\cdot$ N. Alvarez

Laboratoire d'Entomologie Evolutive, Institut de Biologie,

Université de Neuchâtel, Rue Emile-Argand 11,

CP 158, 2009 Neuchâtel, Switzerland

e-mail: yann.triponez@unine.ch

J. B. Jean-Denis

Institut de Chimie, Université de Neuchâtel,

Rue Emile-Argand 11, CP 158, CH-2009 Neuchâtel, Switzerland 
Table 4 Details of the 50 cardenolides and results from the ANOVA on the pairwise genetic distance between populations with individual cardenolides treated as explanatory factors

\begin{tabular}{|c|c|c|c|}
\hline Cardenolide (Retention Time) & Present in $N$ Sites & $F$ Value & $P$ value \\
\hline 4.3 & 7 & 2.68 & 0.1026 \\
\hline 4.8 & $18^{\dagger}$ & - & - \\
\hline 6.5 & $18^{\dagger}$ & - & - \\
\hline 10.6 & $18^{\dagger}$ & - & - \\
\hline 10.8 & 2 & 0.43 & 0.5146 \\
\hline $11.1^{\mathrm{a}}$ & 16 & 7.39 & 0.0068 \\
\hline 11.3 & 13 & 0.01 & 0.9081 \\
\hline 12.0 & $18^{\dagger}$ & - & - \\
\hline 12.8 & 1 & 12.38 & $0.0005 *$ \\
\hline 13.8 & $18^{\dagger}$ & - & - \\
\hline $14.3^{\mathrm{b}}$ & $18^{\dagger}$ & - & - \\
\hline 14.7 & 5 & 0.01 & 0.9388 \\
\hline 15.2 & 9 & 0.04 & 0.8403 \\
\hline 15.9 & 3 & 2.50 & 0.1146 \\
\hline 16.8 & 3 & $<0.01$ & 0.9795 \\
\hline 17.6 & $18^{\dagger}$ & - & - \\
\hline 17.9 & 17 & 20.01 & $<0.0001 *$ \\
\hline $19.0^{\mathrm{c}}$ & 17 & 20.01 & $<0.0001 *$ \\
\hline 19.9 & 12 & 0.01 & 0.9359 \\
\hline 20.5 & 16 & 0.08 & 0.7785 \\
\hline 21.1 & 10 & 1.78 & 0.1828 \\
\hline 21.6 & 17 & 20.01 & $<0.0001 *$ \\
\hline 22.2 & 8 & 0.30 & 0.5870 \\
\hline 22.6 & 7 & 11.97 & $0.0006 *$ \\
\hline 23.1 & 8 & 0.12 & 0.7333 \\
\hline 23.6 & 2 & 13.65 & $0.0002 *$ \\
\hline 24.0 & 14 & 2.67 & 0.1033 \\
\hline $24.4^{\mathrm{d}}$ & $18^{\dagger}$ & - & - \\
\hline 24.7 & 5 & 5.18 & 0.0233 \\
\hline 25.2 & $18^{\dagger}$ & - & - \\
\hline $25.6^{\mathrm{e}}$ & 10 & 22.92 & $<0.0001 *$ \\
\hline 26.6 & 17 & 2.77 & 0.0967 \\
\hline 27.1 & 8 & 1.20 & 0.2742 \\
\hline 27.8 & 11 & 0.05 & 0.8166 \\
\hline 28.3 & 16 & 0.70 & 0.4047 \\
\hline 28.9 & 11 & 3.29 & 0.0705 \\
\hline 29.6 & $18^{\dagger}$ & - & - \\
\hline 30.2 & 4 & 2.02 & 0.1562 \\
\hline 31.2 & 9 & 14.89 & $0.0001 *$ \\
\hline 32.0 & 12 & 0.11 & 0.7425 \\
\hline 32.6 & 12 & 4.00 & 0.0460 \\
\hline $33.0^{\mathrm{f}}$ & $18^{\dagger}$ & - & - \\
\hline $34.1^{\mathrm{g}}$ & $18^{\dagger}$ & - & - \\
\hline 36.6 & 7 & 1.87 & 0.1726 \\
\hline 37.4 & 8 & 1.28 & 0.2593 \\
\hline $37.7^{\mathrm{h}}$ & $18^{\dagger}$ & - & - \\
\hline $38.9^{\mathrm{i}}$ & $18^{\dagger}$ & - & - \\
\hline 39.3 & 3 & 3.55 & 0.0601 \\
\hline
\end{tabular}


Table 4 (continued)

\begin{tabular}{llll}
\hline Cardenolide (Retention Time) & Present in $N$ Sites & $F$ Value & $P$ value \\
\hline 39.9 & 6 & 15.91 & $<0.0001 *$ \\
40.4 & 1 & 2.35 & 0.1258
\end{tabular}

${ }^{\dagger}$ Monomorphic cardenolides: these compounds were found in all populations

*Significant after Bonferroni's correction

a Tetrahydroxylated aglycon-hexopyranoside

${ }^{\mathrm{b}}$ Periplogenin-3-O-[ $\beta$-D-xylopyranosyl- $(1 \rightarrow 4)-\beta-\mathrm{D}$-allopyranoside]

${ }^{\mathrm{c}}$ Tetrahydroxylated aglycon-acetyl-hexopyranoside

${ }^{\mathrm{d}}$ Didehydroperiplogenin-3-O- $\beta$-D-allopyranoside

${ }^{\text {e }}$ Periplogenin-3-O- $\beta$-D-allopyranoside

${ }^{\mathrm{f}}$ Didehydroperiplogenin- $O$-acetyl- $\beta$-D-allopyranoside

${ }^{g}$ Periplogenin-3-O-acetyl- $\beta$-D-allopyranoside

${ }^{\mathrm{h}}$ Didehydrodigitoxigenin-3- $O$-[- $O$-acetyl- $\beta$-D-xylopyranosyl- $(1 \rightarrow 4)-O$-acetyl- $\beta$-D-allopyranoside]

${ }^{\mathrm{i}}$ Digitoxigenin-3-O-[-O-acetyl- $\beta$-D-xylopyranosyl- $(1 \rightarrow 4)$ - $O$-acetyl- $\beta$-D-allopyranoside] 